\title{
How does axial prestretching change the mechanical response of nonlinearly elastic incompressible thin-walled tubes
}

\author{
Lukáš Horný $^{1 *}$, Marek Netušil ${ }^{2}$ \\ ${ }^{1}$ Faculty of Mechanical Engineering, Czech Technical University in Prague, Technická 4, Prague, \\ 16607, Czech Republic, lukas.horny@,fs.cvut.cz \\ ${ }^{2}$ Faculty of Mathematics and Physics, Charles University in Prague, Ke Karlovu 3, Prague, 121 16, \\ Czech Republic, marek.netusil@gmail.com
}

*Corresponding author: Lukáš Horný, Lukas.horny@fss.cvut.cz

\begin{abstract}
Human arteries provide an example of anisotropic, nonlinearly elastic, incompressible tubes. It is known that they operate in situ with significant axial prestretching. In ageing, this prestretching is successively relaxed due to arteriosclerosis. Ex vivo inflation-extension experiments have shown that axial prestretching is advantageous from the mechanical point of view, because it reduces the extent of the axial stress and strain that is experienced by arteries during the heart cycle. It has also recently been found that axial prestretch enhances circumferential deformations. Highly prestretched arteries exhibit higher circumferential stretches than their weakly prestretched counterparts, and this is advantageous when blood is transported to the periphery. However, this effect of axial prestretch on the mechanical response of a tube has until now been overlooked in the scientific literature. The objective of our study is to elucidate the physical cause of this phenomenon. An analytical model of an incompressible thinwalled closed tube was used to simulate the mechanical response of an initially prestretched tube to internal pressure. Four different situations were considered: (I) a hyperelastic material with a large strain stiffening property, (II) a neo-Hookean material, (III) a neo-Hookean material with small strains but large displacements (second order linear elasticity), and (IV) a neo-Hookean material with small strains and small displacements (first order linear elasticity). The results have shown that the positive effect of axial prestretch is not a property exclusively related to anisotropy. It has been proved that nonlinear effects are crucial. Nonlinear constitutive models depending on more than one parameter can both enhance and suppress the circumferential distensibility of the tube due to prestretching. However, a oneparameter neo-Hookean model showed only increased circumferential distensibility. A reduction in second order linear elasticity led to mechanical responses that exhibited only a slight effect of being prestretched. Total linearization proved that axial prestretch has a positive effect only to the point where deformed configuration and reference configuration are distinguished.
\end{abstract}

Keywords: axial prestrain; hyperelasticity; incompressibility; linearization; thin-walled tube; pressurization. 


\section{INTRODUCTION}

An analytical model of a thin-walled tube based on the Laplace law is frequently used in physics and in the engineering sciences to obtain an elementary picture of a mechanical state. In biomechanics, nonlinearly elastic incompressible tubes are used to model arteries, veins, the oesophagus and other tubular organs (Fung 1990, 1997; Humphrey 2002; Taber 2004). The solutions that are obtained are usually considered to be first-order approximations, because the imposed assumptions of the model (the thickness-to-radius ratio, the residual stress and strain, the geometrical non-uniformity etc.; Holzapfel et al., 2000; Holzapfel and Ogden 2010; Horný et al., 2014b) are imperfectly satisfied. The simplicity of the thin-walled tube model might induce the impression that our knowledge of its mechanical response is exhaustive. In this study, however, we will show an example of a phenomenon that has been overlooked until now: the enhanced circumferential distensibility of a pressurized tube due to initial axial prestretching.

Human arteries in situ are significantly prestretched in the axial direction (this was probably first reported in the context of biomechanics by Fuchs in 1900, as mentioned by Bergel (1961)). This prestretching is observed during an autopsy as a retraction of the excised arterial segment (Horný et al, 2011, 2012, 2013, 2014a), and the prestretch $\lambda_{z Z}{ }^{i n i}$ is defined as the ratio of the in situ-to-ex situ length of the segment. Ex vivo inflation-extension experiments have shown that axial prestretching is advantageous from the mechanical point of view, because it reduces the extent of the axial stress and strain that is experienced by arteries during the heart cycle (Dobrin and Doyle, 1970). In the optimal case of a young and healthy individual, there is a certain prestretch value at which the artery can be pressurized without a significant change to its length, so it can transmit a pressure pulse wave with negligible axial deformation (Van Loon et al., 1977; Schulze-Bauer et al., 2003; Sommer et al., 2010).

However, recent studies by Horný et al. $(2011,2012,2013,2014 b)$ have shown that ageing of the cardiovascular system is, besides general stiffening of elastic arteries, also manifested by a reduction of axial prestretch. Nevertheless, a detailed analysis of the constitutive behaviour of 17 human aortas suggested that aged aortas, although weakly prestretched, still can benefit from the remaining prestretch (Horný et al., 2014b). The decreasing of the prestretch is individual process similarly to (perhaps better to say as a consequence of) the progress of human ageing. A statistical variability reported in Horny et al. (2014b) implies that, for example, a 60-year-old man has the expected axial prestretch $\lambda_{z Z}{ }^{i n i}=1.08$ with a $95 \%$ confidence interval for a prediction $\lambda_{z Z} z^{i n i} \in[1.00 ; 1.16]$. An analytical simulation of the inflation-extension response showed that, depending on the initial prestretch, the abdominal aorta of a 60 -year-old man sustains the following changes in axial stretch $\lambda_{z Z}\left(P_{\text {SYSTOLE }}\right)-\lambda_{z Z}\left(P_{\text {DIASTOLE }}\right)=0.016$, 0.003 , and 0.025 for $\lambda_{z z}{ }^{i n i}=1.08,1.16$, and 1.00, respectively (Horný et al., 2014b). The corresponding normalized changes in the axial Cauchy stress $\left(\sigma_{z z}\left(P_{\text {SYstole }}\right)-\sigma_{z z}\left(P_{\text {Diastole }}\right)\right) / \sigma_{z z}\left(P_{\text {SYSTOLE }}\right)$ were 0.604 for expected prestretch $\lambda_{z Z} z^{i n i}=1.08,0.426$ for the upper confidence limit of the prestretch $\lambda_{z Z}{ }^{i n i}=1.16$, and 0.769 for the lower limit $\lambda_{z Z}{ }^{i n i}=1.00$. This clearly demonstrates that, although axial prestretch declines (the expected prestretch of the abdominal aorta for a 20 -year-old man is 1.34 , with a $95 \%$ confidence interval for the prediction [1.24;1.43]), remaining prestretch still retains its biomechanical role: to minimize the axial stretch and stress variation. 
Horný et al. (2014b) have moreover shown that axial prestretching also has a significant effect on the circumferential stretch variation $\lambda_{\theta \Theta}\left(P_{\text {SYSTOLE }}\right)-\lambda_{\theta \Theta}\left(P_{\text {DIASTOLE }}\right)$ exhibited during pressurization. Unlike the axial stretch and stress variations, which are minimized by prestretching, circumferential stretch variations were found to be increased by prestretching. For the same example as before of a $60-$ year-old man, the circumferential stretch variation $\lambda_{\theta \Theta}\left(P_{\text {SYSTOLE }}\right)-\lambda_{\theta \Theta}\left(P_{\text {DiAStOLE }}\right)$, which we will refer to here as distensibility, was computed to be 0.059 for $\lambda_{z Z}{ }^{i n i}=1.08 ; 0.067$ for $\lambda_{z Z}{ }^{i n i}=1.16$; and 0.056 for $\lambda_{z Z} z^{i n i}=1.00$. The study conducted by Horný et al. (2014b) revealed this phenomenon for all 17 investigated aortas. Higher axial prestretching induced inflation responses exhibiting higher circumferential distensibility of the tubes. This implies that the arterial physiology benefits in two ways from prestretching. The first way is from minimization of the axial stress and strain variation during the heart cycle, and the second way is from maximization of the circumferential distensibility during the cycle. Since arteries are conduits for the flowing blood, the higher distensibility of prestretched arteries means that they can accommodate a greater volume of blood at the same pressure than their nonprestretched counterparts. This leads us to regard the effect of axial prestretching as positive. To the best of our knowledge, this is the first time that such a conclusion on the effect on circumferential distensibility described in Horný et al. (2014b) has been presented in the literature.

In the authors' opinion, the positive effect of axial prestretching on circumferential distensibility is rather contra-intuitive at first sight, because we might expect a nonlinearly elastic tube to reach a stiffer state when pretension is applied. In their study, Horný et al. (2014b) hypothesized that anisotropy may be responsible for this phenomenon, because the elastic artery wall is reinforced by helically aligned collagen fibres (Holzapfel et al., 2000; Gasser et al. 2006; Horný et al., 2009, 2010) and Horný et al. (2014b) did indeed use an anisotropic constitutive model. However, they did not compare their results with the mechanical response of isotropic tubes, and anisotropy as a cause of the phenomenon remained only a hypothesis.

An objective of our paper is to show what physical mechanism is responsible for the enhancement the circumferential distensibility of an inflated tube. A bottom-up approach will be used to demonstrate what happens. The model of an incompressible nonlinearly elastic thin-walled tube will be simplified step-by-step from a material with exponential elastic potential at large strains to a linearly elastic material at small strains, and the cause of the enhanced circumferential distensibility will be made clear. We can state in advance that a problem formulated with large displacements but small strains for a linear material (second order linear elasticity) exhibits enhanced circumferential distensibility, and in the elementary linear elasticity of small displacements the model shows no positive effect of axial prestretch.

\section{MeTHODS}

Two different analytical models were used in Horný et al. (2014b). These were the thick-walled computational model, which is capable of accounting for residual strains, and the thin-walled model, which operates with averaged stresses acting on mid-surface of the tube. As is documented in that paper, the two models, although they differ numerically, give the same qualitative result - axial prestrain enhances circumferential distensibility. Since the effect of prestretching is captured by both models, in 
what follows, for the sake of simplicity and for clear and easy interpretation of the results, only the thinwalled model will be of our interest.

Consider a long thin-walled cylindrical tube with closed ends that, in the reference configuration, has middle radius $R$, thickness $H$, and length $L$. Assume that, during pressurization, the motion of the material particle located originally at $(R, \Theta, Z)$, which is sufficiently distant from ends, is described by the equations summarized in (1).

$r=\lambda_{\theta \Theta} R, \quad h=\lambda_{r R}, \quad z=\lambda_{z Z} Z, \quad \theta=\Theta$

Here $r$ denotes the deformed middle radius and $h$ denotes thickness. Equations (1) express the fact that the tube inflates and extends (or shortens) uniformly, and that it does not twist. The stretches $\lambda_{k K}(k=r, \theta, z ; K=R, \Theta, Z)$ are the components of the deformation gradient $\mathbf{F}, \mathbf{F}=\operatorname{diag}\left[\lambda_{r R}, \lambda_{\theta \Theta}, \lambda_{z Z}\right]$. The right Cauchy-Green strain tensor $\mathbf{C}$ and Green-Lagrange strain tensor $\mathbf{E}$ can be computed as $\mathbf{C}=$ $\mathbf{F}^{\mathrm{T}} \mathbf{F}$ and $\mathbf{E}=1 / 2(\mathbf{C}-\mathbf{I})$, where $\mathbf{I}$ is a second-order unit tensor. The material of the tube is considered to be incompressible, so the volume ratio $J, J=\operatorname{det}(\mathbf{F})$, gives equation (2) expressing $J=1$.

$\lambda_{z Z} \lambda_{r R} \lambda_{\Theta \Theta}=1$

The equilibrium equations of a thin-walled tube with closed ends initially prestretched by axial force $F_{r e d}$ and loaded by internal pressure $P$ can be written in the form (3). Here $\sigma_{r r}, \sigma_{\theta \theta}$, and $\sigma_{z z}$ denote the radial, circumferential and axial component, respectively, of the Cauchy stress tensor $\sigma$.

$\sigma_{r r}=-\frac{P}{2}, \quad \sigma_{\theta \theta}=\frac{P r}{h}, \quad \sigma_{z z}=\frac{P r}{2 h}+\frac{F_{r e d}}{2 \pi r h}$

The material of the tube is considered to be hyperelastic, described by the strain energy density function (elastic potential) $W$ defined per unit reference volume. In this case, the constitutive equation relating components of the stress and strain tensor can be written in the form of (4). Here $p$ denotes a Lagrangean multiplier reflecting the hydrostatic stress contribution (not captured in $W$, due to incompressibility) which has to be determined from the force boundary condition.

$\sigma_{r r}=\lambda_{r R} \frac{\partial W}{\partial \lambda_{r R}}-p, \quad \sigma_{\theta \theta}=\lambda_{\theta \Theta} \frac{\partial W}{\partial \lambda_{\theta \Theta}}-p, \quad \sigma_{z z}=\lambda_{z Z} \frac{\partial W}{\partial \lambda_{z Z}}-p$

Equations governing the inflation and extension of the thin-walled tube are obtained after substituting (5) into (3). The system is given explicitly in (6).

$\lambda_{r R} \frac{\partial W}{\partial \lambda_{r R}}-p=-\frac{P}{2}, \quad \lambda_{\theta \Theta} \frac{\partial W}{\partial \lambda_{\theta \Theta}}-p=\frac{P r}{2 h}, \quad \lambda_{z Z} \frac{\partial W}{\partial \lambda_{z Z}}-p=\frac{P r}{2 h}+\frac{F_{r e d}}{2 \pi r h}$

For the material behaviour, Horný et al. (2014b) modelled the artery as an anisotropic material described by the Fung-type elastic potential $W_{G M W}$ (7), which was introduced in Guccione et al. (1991). Here $c_{0}$ is a stress-like material parameter. $c_{1}$ and $c_{2}$ are dimensionless parameters which govern the anisotropy of the material. $E_{K K}(K=R, \Theta, Z)$ are components of the Green-Lagrange strain tensor expressed in the cylindrical coordinate system. 
$W_{G M W}=\frac{c_{0}}{2}\left(e^{c_{1} E_{\Theta \Theta}^{2}+c_{2}\left(E_{Z Z}^{2}+E_{R R}^{2}\right)}-1\right)$

In what follows, four different cases will be investigated. They are: (I) an isotropic nonlinearly elastic thin-walled tube described by the strain energy density function exhibiting large strain stiffening studied at finite strains; (II) a neo-Hookean tube at finite strains; (III) a linearized neo-Hookean tube studied at small strains but large displacements (second order linear elasticity); and (IV) a linearized neo-Hookean tube studied at small strains and small displacements (first order linear elasticity).

\subsection{ISOTROPIC LARGE STRAIN STIFFENING MODEL}

Since Horný et al. (2014b) documented the positive effect of axial prestretching in an anisotropic material, we will now show whether it is preserved when the problem is reduced to isotropy. The potential (7) belongs to the class of so-called Fung-type models (Humphrey 2002). This is a family of elastic potentials based on the exponential function, which has been proved to be very successful in describing the mechanical behaviour of soft tissues (arteries, veins, myocardium, skin, tendons, and ligaments), which generally exhibit large strain stiffening attributed to gradual load-bearing engagement of collagen fibres (Holzapfel et al., 2000; Holzapfel and Ogden, 2010). The first representative of this family was introduced by Y.C. Fung; Fung (1967), and Fung et al. (1979). The simplest isotropic representative of this family is the Fung-Demiray model $W_{F D}(8)$, which was proposed in Demiray (1972).

$W_{F D}=\frac{\mu}{2 \beta}\left(e^{\beta\left(I_{1}-3\right)}-1\right)$

Here $\mu$ is a stress-like parameter which at infinitesimal strains corresponds to the shear modulus. $\beta$ is a dimensionless parameter modulating the rate of strain stiffening. $I_{1}$ is the first principal invariant of $\mathbf{C}$ and is expressed in (9). In the cylindrical coordinate system and under the kinematics adopted for an inflated-extended thin-walled tube in (1), equations (6) with substituted (8) and (9) have the form of (10-12).

$$
\begin{aligned}
& I_{1}=\lambda_{r R}^{2}+\lambda_{\theta \Theta}^{2}+\lambda_{z Z}^{2} \\
& \mu \lambda_{r R}^{2} e^{\beta\left(\lambda_{r R}^{2}+\lambda_{\theta \Theta}^{2}+\lambda_{z Z}^{2}-3\right)}-p=-\frac{P}{2} \\
& \mu \lambda_{\theta \Theta}^{2} e^{\beta\left(\lambda_{r R}^{2}+\lambda_{\theta \Theta}^{2}+\lambda_{z Z}^{2}-3\right)}-p=\frac{r P}{h} \\
& \mu \lambda_{z Z}^{2} e^{\beta\left(\lambda_{r R}^{2}+\lambda_{\Theta \Theta}^{2}+\lambda_{z Z}^{2}-3\right)}-p=\frac{F_{r e d}}{2 \pi r h}+\frac{r P}{2 h}
\end{aligned}
$$


The Lagrangean multiplier $p$, which accounts for the hydrostatic stress contribution, is determined from (10). This is substituted into (11) and (12). The incompressibility condition (2) and geometric equations (1) are subsequently used to obtain the final form of the governing equations (13-14).

$$
\begin{aligned}
& \mu e^{\beta\left(\frac{1}{\lambda_{\theta}^{2} \lambda_{z Z}^{2}}+\lambda_{\oplus \Theta}^{2}+\lambda_{z Z}^{2}-3\right)}\left(\lambda_{\theta \Theta}^{2}-\frac{1}{\lambda_{\theta \Theta}^{2} \lambda_{z Z}^{2}}\right)+\frac{P}{2}=\lambda_{\theta \Theta}^{2} \lambda_{z Z} \frac{R P}{H} \\
& \mu e^{\beta\left(\frac{1}{\lambda_{\oplus \Theta}^{2} \lambda_{z Z}^{2}}+\lambda_{\theta \Theta}^{2}+\lambda_{z Z}^{2}-3\right)}\left(\lambda_{z Z}^{2}-\frac{1}{\lambda_{\theta \Theta}^{2} \lambda_{z Z}^{2}}\right)+\frac{P}{2}=\lambda_{\theta \Theta}^{2} \lambda_{z Z} \frac{R P}{H}+\lambda_{z Z} \frac{F_{r e d}}{2 \pi R H}
\end{aligned}
$$

Before we proceed to solve (13-14), the equations will be converted to dimensionless form by (a) dividing by $\mu$, (b) introducing the aspect ratio $\varepsilon=H / R$, and (c) introducing the dimensionless pressure $P_{\mu}=P / \mu$ and the dimensionless force $F_{\mu}=F_{\text {red }} /\left(\pi R^{2} \mu\right)$. The system that is obtained is given in (15) and (16).

$$
\begin{aligned}
& e^{\beta\left(\frac{1}{\lambda_{\Theta}^{2} \lambda_{z Z}^{2}}+\lambda_{\Theta \Theta}^{2}+\lambda_{z Z}^{2}-3\right)}\left(\lambda_{\Theta \Theta}^{2}-\frac{1}{\lambda_{\Theta \Theta}^{2} \lambda_{z Z}^{2}}\right)-\frac{P_{\mu}}{2}=\frac{P_{\mu}}{\varepsilon} \lambda_{\Theta \Theta}^{2} \lambda_{z Z} \\
& e^{\beta\left(\frac{1}{\lambda_{\Theta \Theta}^{2} \lambda_{z Z}^{2}}+\lambda_{\Theta \Theta}^{2}+\lambda_{z Z}^{2}-3\right)}\left(\lambda_{z Z}^{2}-\frac{1}{\lambda_{\Theta \Theta}^{2} \lambda_{z Z}^{2}}\right)-\frac{P_{\mu}}{2}=\frac{F_{\mu}}{2 \varepsilon} \lambda_{z Z}+\frac{P_{\mu}}{\varepsilon} \lambda_{\Theta \Theta}^{2} \lambda_{z Z}
\end{aligned}
$$

It is clear that $P_{\mu}$ and $F_{\mu}$ can easily be resolved from (15-16) as $P_{\mu}=P_{\mu}\left(\lambda_{\theta \Theta}, \lambda_{z z}\right)$ and $F_{\mu}=F_{\mu}\left(\lambda_{\theta \Theta}, \lambda_{z z}\right)$. However, when circumferential distensibility is treated, we are much more interested in $\lambda_{\theta \Theta}=\lambda_{\theta \Theta}\left(P_{\mu}, F_{\mu}\right)$ and $\lambda_{z z}=\lambda_{z z}\left(P_{\mu}, F_{\mu}\right)$. Since (15-16) is nonlinear in $\lambda_{\theta \Theta}$ and $\lambda_{z z}$, we will continue with a numerical solution. This was conducted in Maple 18, using the $f$ solve command, choosing axial prestretching $\lambda_{z} z^{i n i} \in\{0.1(i-1)+1\}_{i=1^{n}}^{n=11}$, computing $F_{\mu}$ and $\lambda_{\theta \Theta^{i n i}}$ at $P_{\mu}=0$, and finally solving (1516) at a given $F_{\mu}$ and $\lambda_{\theta \Theta} \in\left\{0.001(i-1)+\lambda_{\theta \Theta} \Theta^{n i}\right\}_{i=1}{ }^{n}=1500$ for unknown $P_{\mu}$ and $\lambda_{z z}$. In the representative example, $\beta=1$ was prescribed.

\subsection{NEO-HOOKEAN MODEL AT FINITE STRAINS}

Strain energy density models (7) and (8) are exponential functions of deformation, and they exhibit rapid large strain stiffening (Kanner and Horgan, 2007; Horgan and Saccomandi, 2003; Horgan, 2015; Ogden and Saccomandi, 2007; Horný et al., 2014c). Depending on specific values of the material parameters, the materials described by these potentials are characterized by progressively increasing stress-strain relationships, which is typical for soft biological tissues. As the second case, rapid strain stiffening will be suppressed, and the procedure will be repeated with the simplest invariant-based nonlinear material model (17). This is the so-called neo-Hooke strain energy density function which, under moderate strains, creates a link between the phenomenological theory and the statistical theory of macromolecular materials (Holzapfel, 2000). 


$$
W_{n H}=\frac{\mu}{2}\left(I_{1}-3\right)
$$

Here $\mu$ is a stress-like material parameter which at infinitesimal strains corresponds to the shear modulus. The mutual relationship between $W_{n H}$ and $W_{F D}$ is given by (18).

$\lim _{\beta \rightarrow 0} \frac{\mu}{2 \beta}\left(e^{\beta\left(I_{1}-3\right)}-1\right)=\frac{\mu}{2}\left(I_{1}-3\right)$

The constitutive equations obtained by substituting (17) into (5) are listed in (19). It can be observed that the material nonlinearity (strain stiffening) is lacking here, because $\lambda_{k K^{2}}(k=r, \theta, z ; K=R, \Theta, Z)$ represents geometrical nonlinearity.

$$
\sigma_{r r}=\mu \lambda_{r R}^{2}-p \quad \sigma_{\theta \theta}=\mu \lambda_{\theta \Theta}^{2}-p \quad \sigma_{z z}=\mu \lambda_{z Z}^{2}-p
$$

(19) is substituted into the equilibrium equations (3) in (20-22). (20) determines $p$.

$$
\begin{aligned}
& \mu \lambda_{r R}^{2}-p=-\frac{P}{2} \\
& \mu \lambda_{\Theta \Theta}^{2}-p=\frac{r P}{h} \\
& \mu \lambda_{z z}^{2}-p=\frac{F_{r e d}}{2 \pi r h}+\frac{r P}{2 h}
\end{aligned}
$$

Substituting $p$ and applying geometrical equations (1), the system (23-24) governing the inflationextension response of the thin-walled tube is obtained.

$$
\begin{aligned}
& \mu\left(\lambda_{\Theta \Theta}^{2}-\frac{1}{\lambda_{\theta \Theta}^{2} \lambda_{z Z}^{2}}\right)-\frac{P}{2}=\frac{P R}{H} \lambda_{\Theta \Theta}^{2} \lambda_{z Z} \\
& \mu\left(\lambda_{z Z}^{2}-\frac{1}{\lambda_{\theta \Theta}^{2} \lambda_{z Z}^{2}}\right)-\frac{P}{2}=\frac{F_{r e d}}{2 \pi R H} \lambda_{z Z}+\frac{P R}{2 H} \lambda_{\Theta \Theta}^{2} \lambda_{z Z}
\end{aligned}
$$

Finally, the aspect ratio $\varepsilon=H / R$, dimensionless pressure $P_{\mu}=P / \mu$, and dimensionless force $F_{\mu}=F_{\text {red }} /\left(\pi R^{2}\right) / \mu$ are again introduced in (25-26).

$$
\begin{aligned}
& \lambda_{\theta \Theta}^{2}-\frac{1}{\lambda_{\Theta \Theta}^{2} \lambda_{z Z}^{2}}-\frac{P_{\mu}}{2}=\frac{P_{\mu}}{\varepsilon} \lambda_{\Theta \Theta}^{2} \lambda_{z Z} \\
& \lambda_{z Z}^{2}-\frac{1}{\lambda_{\Theta \Theta}^{2} \lambda_{z Z}^{2}}-\frac{P_{\mu}}{2}=\frac{F_{\mu}}{2 \varepsilon} \lambda_{z Z}+\frac{P_{\mu}}{2 \varepsilon} \lambda_{\Theta \Theta}^{2} \lambda_{z Z}
\end{aligned}
$$

We observe that equations (25-26) differ from (15-16) only by the absence of the exponential term, which is in accordance with (18). Since (25-26) are again nonlinear with respect to $\lambda_{\theta \Theta}$ and $\lambda_{z z}$, the same approach as in the case of the exponential model will be employed to obtain the extension-inflation behaviour of a neo-Hookean cylindrical tube. 


\subsection{SECOND ORDER LINEAR ELASTICITY (SMALL STRAINS BUT LARGE DISPLACEMENTS)}

In this section, nonlinear effects will be attenuated by a transition from finite strain theory to linearized elasticity at infinitesimal strains. First, let us reconsider the constitutive equations implied by the neoHooke material model (19). Note that the left sides of (23-24) express $\sigma_{\theta \theta}$ and $\sigma_{z z}$ after $p$ is substituted from the radial equilibrium. Similarly, $\sigma_{\theta \theta} / \mu$ and $\sigma_{z z} / \mu$ are given by the left sides in (25-26). They are repeated in (27-28). It is clear that incompressibility at finite strains is manifested in the constitutive equations by “ $-1 /\left(\lambda_{\theta \Theta}{ }^{2} \lambda_{z z^{2}}\right)-P_{\mu} / 2$."

$$
\begin{gathered}
\frac{\sigma_{\theta \theta}}{\mu}=\lambda_{\theta \Theta}^{2}-\frac{1}{\lambda_{\theta \Theta}^{2} \lambda_{z Z}^{2}}-\frac{P_{\mu}}{2} \\
\frac{\sigma_{z z}}{\mu}=\lambda_{z Z}^{2}-\frac{1}{\lambda_{\theta \Theta}^{2} \lambda_{z Z}^{2}}-\frac{P_{\mu}}{2}
\end{gathered}
$$

We will now start from (19), which will be linearized. As the first step, the stretches $\lambda_{k K}$ in (19) are interchanged by components of the Green-Lagrange strain tensor $E_{K K}=1 / 2\left(\lambda_{k K}{ }^{2}-1\right),(29)$.

$$
\sigma_{r r}=\mu\left(2 E_{R R}+1\right)-p, \quad \sigma_{\theta \theta}=\mu\left(2 E_{\Theta \Theta}+1\right)-p, \quad \sigma_{z z}=\mu\left(2 E_{Z Z}+1\right)-p
$$

At this point, a description by the infinitesimal (engineering) strain tensor $\varepsilon$ is introduced into (29). Since $\mathbf{E}$ and $\varepsilon$ are approximately equal in the range of infinitesimal theory, we simply interchange $E_{K K}$ and $\varepsilon k k$, obtaining (30) from (29).

$$
\sigma_{r r}=\mu\left(2 \varepsilon_{r r}+1\right)-p \quad \sigma_{\theta \theta}=\mu\left(2 \varepsilon_{\theta \theta}+1\right)-p \quad \sigma_{z z}=\mu\left(2 \varepsilon_{z z}+1\right)-p
$$

It is clear from (30a) that (31) holds.

$$
p=\mu\left(2 \varepsilon_{r r}+1\right)+\frac{P}{2}
$$

We also need to express geometric equations (1) by means of $\varepsilon$. This is done in system (32).

$$
h=\left(1+\varepsilon_{r r}\right) H \quad r=\left(1+\varepsilon_{\theta \theta}\right) R \quad z=\left(1+\varepsilon_{z z}\right) Z
$$

The incompressibility condition for infinitesimal strains can be written in the form $\varepsilon_{r r}+\varepsilon_{\theta \theta}+\varepsilon_{z z}=0$. Hence, the radial component of the engineering strain tensor can be substituted by (33).

$\varepsilon_{r r}=-\varepsilon_{\theta \theta}-\varepsilon_{z z}$

Substituting (31) into (30b) and (30c), applying (32) and substituting all into the equilibrium equations, system (34-35) is obtained. 


$$
\begin{aligned}
& \mu\left(2 \varepsilon_{\theta \theta}+1\right)-\mu\left(1-2 \varepsilon_{\theta \theta}-2 \varepsilon_{z z}\right)-\frac{P}{2}=\frac{\left(1+\varepsilon_{\theta \theta}\right) R P}{\left(1-\varepsilon_{\theta \theta}-\varepsilon_{z z}\right) H} \\
& \mu\left(2 \varepsilon_{z z}+1\right)-\mu\left(1-2 \varepsilon_{\theta \theta}-2 \varepsilon_{z z}\right)-\frac{P}{2}=\frac{1}{\left(1+\varepsilon_{\theta \theta}\right)\left(1-\varepsilon_{\theta \theta}-\varepsilon_{z z}\right)} \frac{F_{r e d}}{2 \pi R H}+\frac{\left(1+\varepsilon_{\theta \theta}\right) R P}{2\left(1-\varepsilon_{\theta \theta}-\varepsilon_{z z}\right) H}
\end{aligned}
$$

Equations (34-35) govern the inflation-extension response of a thin-walled incompressible linearly elastic tube in so-called second order linear elasticity theory. This means that although the components of the small strain tensor are present in the equations, we still distinguish between the deformed configuration and the reference configuration. Thus equations (34-35) express the equality between the Cauchy stresses computed from the constitutive equations (left sides) and the Cauchy stresses computed from the geometry and the loads (right sides). The situation is similar to the way in which the buckling of a compressed column is treated. To obtain the critical force from a discussion of the boundary conditions, one has to substitute the expression for the bending moment into the equation for the deflection of the beam (Euler-Bernoulli) from the internal reaction forces determined in the deformed configuration. In other words, small strains with large displacements are considered here.

Applying the same normalization procedure as in the previous cases, system (34-35) is transformed into dimensionless form (36-37).

$$
\begin{aligned}
& 2\left(2 \varepsilon_{\theta \theta}+\varepsilon_{z z}\right)-\frac{P_{\mu}}{2}=\frac{1+\varepsilon_{\theta \theta}}{1-\varepsilon_{\theta \theta}-\varepsilon_{z z}} \frac{P_{\mu}}{\varepsilon} \\
& 2\left(2 \varepsilon_{z z}+\varepsilon_{\theta \theta}\right)-\frac{P_{\mu}}{2}=\frac{1}{\left(1+\varepsilon_{\theta \theta}\right)\left(1-\varepsilon_{\theta \theta}-\varepsilon_{z z}\right)} \frac{F_{\mu}}{2 \varepsilon}+\frac{1+\varepsilon_{\theta \theta}}{1-\varepsilon_{\theta \theta}-\varepsilon_{z z}} \frac{P_{\mu}}{2 \varepsilon}
\end{aligned}
$$

The final equations (36-37) remain nonlinear, because rational expressions occur here. The nonlinearity, however, comes solely from the large displacements (the rational expressions are on the right sides of the equations). The results will again be obtained numerically.

It is interesting to see how the equations are expressed by means of the components the GreenLagrange strain tensor and finite strain theory. The finite strain counterparts of (36-37) are obtained from (25-26) by transforming $\lambda_{k K}$ into $E_{K K}$. They are given in (38) and (39).

$$
\begin{aligned}
& 2 E_{\Theta \Theta}+1-\frac{1}{\left(2 E_{\Theta \Theta}+1\right)\left(2 E_{\mathrm{ZZ}}+1\right)}-\frac{P_{\mu}}{2}=\frac{P_{\mu}}{\varepsilon}\left(2 E_{\Theta \Theta}+1\right) \sqrt{2 E_{\mathrm{ZZ}}+1} \\
& 2 E_{\mathrm{ZZ}}+1-\frac{1}{\left(2 E_{\Theta \Theta}+1\right)\left(2 E_{\mathrm{ZZ}}+1\right)}-\frac{P_{\mu}}{2}=\frac{F_{\mu}}{2 \varepsilon} \sqrt{2 E_{\mathrm{ZZ}}+1}+\frac{P_{\mu}}{2 \varepsilon}\left(2 E_{\Theta \Theta}+1\right) \sqrt{2 E_{\mathrm{ZZ}}+1}
\end{aligned}
$$

The left sides of (38-39) give equations for $\sigma_{\theta \theta} / \mu$ and $\sigma_{z z} / \mu$ when $p$ has been eliminated. In (40-42), these equations are rewritten into the form of constitutive equations. Note that incompressibility necessitates loads to enter into the equations. Hence equations (40-42) are not general but they are valid only for the pressurization of a thin-walled tube. 


$$
\begin{aligned}
\sigma_{r r} & =-\frac{P}{2} \\
\sigma_{\theta \theta} & =\mu\left(2 E_{\Theta \Theta}+1\right)-\frac{\mu}{\left(2 E_{\Theta \Theta}+1\right)\left(2 E_{\mathrm{ZZ}}+1\right)}-\frac{P}{2} \\
\sigma_{z z} & =\mu\left(2 E_{\mathrm{ZZ}}+1\right)-\frac{\mu}{\left(2 E_{\Theta \Theta}+1\right)\left(2 E_{\mathrm{ZZ}}+1\right)}-\frac{P}{2}
\end{aligned}
$$

It is clear that " $\mu /\left(2 E_{\Theta \Theta}+1\right) /\left(2 E_{z z}+1\right)-1 / 2 P$ " arises from incompressibility. We can compare (40-42) with (43-45). (43-45) are again constitutive equations obtained from the left sides of (34-35), which are valid for the specific case of a linearly elastic incompressible thin-walled tube with $\sigma_{r r}$ determined from the external load. It is clear that a correct description using finite strains generates nonlinearity in (41-42) via the incompressibility condition, which is lacking in the description based on the infinitesimal strain tensor (44-45); “ $\mu /\left(2 E_{\Theta \Theta}+1\right) /\left(2 E_{z z}+1\right)$ ” vs. " $\mu\left(1-2 \varepsilon_{\theta \theta}-2 \varepsilon_{z z}\right)$ ”.

$$
\begin{aligned}
& \sigma_{r r}=-\frac{P}{2} \\
& \sigma_{\theta \theta}=\mu\left(2 \varepsilon_{\theta \theta}+1\right)-\mu\left(1-2 \varepsilon_{\theta \theta}-2 \varepsilon_{z z}\right)-\frac{P}{2} \\
& \sigma_{z z}=\mu\left(2 \varepsilon_{z z}+1\right)-\mu\left(1-2 \varepsilon_{\theta \theta}-2 \varepsilon_{z z}\right)-\frac{P}{2}
\end{aligned}
$$

\subsection{FIRST ORDER LINEAR ELASTICITY (SMALL STRAINS AND DISPLACEMENTS)}

Total linearization involves (a) introducing the small strain tensor, (b) linearizing the constitutive equations, and (c) taking into consideration small displacements, which justifies substituting the nominal stress tensor (the current force per reference cross-section) into the equilibrium equations. The constitutive equations are the same as in the previous section, given by (30). Equation (31) is again used to determine the contribution of the hydrostatic stress $p$. Thus the equilibrium equations are given by (34-35), but the right sides are modified according to the assumption of small displacements. The resulting equations are (46-47).

$$
\begin{aligned}
& 2 \mu\left(2 \varepsilon_{\theta \theta}+\varepsilon_{z z}\right)-\frac{P}{2}=\frac{R P}{H} \\
& 2 \mu\left(2 \varepsilon_{z z}+\varepsilon_{\theta \theta}\right)-\frac{P}{2}=\frac{F_{r e d}}{2 \pi R H}+\frac{R P}{2 H}
\end{aligned}
$$


The dimensionless counterparts of (46-47) are equations (48-49).

$2\left(2 \varepsilon_{\theta \theta}+\varepsilon_{z z}\right)-\frac{P_{\mu}}{2}=\frac{P_{\mu}}{\varepsilon}$

$2\left(2 \varepsilon_{z z}+\varepsilon_{\theta \theta}\right)-\frac{P_{\mu}}{2}=\frac{F_{\mu}}{2 \varepsilon}+\frac{P_{\mu}}{2 \varepsilon}$

The first order linear elasticity gives the linear system of the equations of the problem (48-49), and at this moment the explicit dependence of $\varepsilon_{\theta \theta}$ and $\varepsilon_{z z}$ on pressure and force is finally found (50).

$\varepsilon_{\theta \theta}=\frac{1}{12} \frac{P_{\mu}(\varepsilon+3)-F_{\mu}}{\varepsilon} \quad \varepsilon_{z z}=\frac{1}{12} \frac{\varepsilon P_{\mu}+2 F_{\mu}}{\varepsilon}$

Since axial prestrain $\varepsilon_{z z}{ }^{i n i}$ is applied before the pressurization, i.e. at $P_{\mu}=0,(50 \mathrm{~b})$ gives (51). Computed $F_{\mu}$ is constant in the subsequent pressurization. Substituting from (51) into (50a), the explicit dependence of the circumferential strain $\varepsilon_{\theta \theta}$ on the initial axial prestrain $\varepsilon_{z z} z^{i n i}$ is obtained (52).

$\varepsilon_{z z}^{i n i}=\frac{F_{\mu}}{6 \varepsilon}$

$\varepsilon_{\theta \theta}=\frac{1}{12} \frac{\varepsilon+3}{\varepsilon} P_{\mu}-\frac{1}{2} \varepsilon_{z z}^{i n i}$

Equation (52) implies that the axial prestrain will cause nothing more than a shift of the line that represents the $\varepsilon_{\theta \theta}-P_{\mu}$ dependence. However, the slope of the lines is constant during pressurization; from (52), the slope is $(\varepsilon+3) /(12 \varepsilon)$. In contrast to previous cases, the first order linear elasticity immediately shows that in this theory axial prestretching does not affect the character of the pressurization of a thinwalled incompressible tube. It only changes initial conditions of the pressurization. 


\section{NUMERICAL SIMULATIONS, AND A DISCUSSION OF THE RESULTS}

The analytical computational models used in deriving the equations governing the inflation-extension of a closed incompressible thin-walled tube revealed that for (I), the exponential elastic potential (nonlinear model with rapid strain stiffening) - equations (15-16), for (II), a neo-Hookean material at finite strains (nonlinear model with moderate material nonlinearity) - equations (25-26), and for (III), a linearized neo-Hookean material considered at small strains but large displacements - equations (3637), the problem does not lead to systems of equations from which the explicit analytical dependence of the circumferential stretch on the initial axial prestretching can be found. In these cases, numerical simulations were conducted to demonstrate the mechanical behaviour predicted by the models. They were performed in Maple 18, using the fsolve command according to the following scheme:

(I) and (II)

(a) $\lambda z z^{i n i} \in\{1+0.1(i-1)\}_{i=1}^{n=11}$

(b) the prestretching axial force $F_{\mu}$ and the initial circumferential stretch $\lambda_{\theta \Theta}{ }^{i n i}$ were computed for $P_{\mu}=$ 0 and the chosen prestretch $\lambda_{z} z^{i n i}$

(c) $P_{\mu}$ and $\lambda_{z Z}$ are computed for $F_{\mu}$ determined in (b) and $\lambda_{\theta \Theta} \in\left\{\lambda_{\theta \Theta}{ }^{i n i}+0.001(i-1)\right\}^{i=1}{ }^{m}$ where $m=$ 1200 ;

(a) $\varepsilon_{z z^{i n i}} \in\{0.02(i-1)\}_{i=1^{n}}=11$

(b) the prestretching axial force $F_{\mu}$ and the initial circumferential strain $\varepsilon_{\theta \theta}{ }^{\text {ini }}$ were computed for $P_{\mu}=0$ and chosen prestretch $\varepsilon z z^{i n i}$

(c) $P_{\mu}$ and $\varepsilon_{z z}$ are computed for $F_{\mu}$ determined in (b) and $\varepsilon_{\theta \theta} \in\left\{\varepsilon_{\theta \theta}{ }^{i n i}+0.001(i-1)\right\}_{i=1}^{m}$ where $m=1200$.

A thickness-to-radius ratio of $\varepsilon=0.1$ was applied in all the simulations. Parameter $\beta$, modulating the rate of stiffening in (8), was prescribed to be $\beta=1$.

\subsection{RESULTS FOR THE ISOTROPIC LARGE STRAIN STIFFENING MODEL}

Figure 1 depicts the results of the simulation of the inflation-extension behaviour of an incompressible thin-walled tube with isotropic exponential elastic potential (8). Panels A and B show the initial conditions of the inflation, i.e. the dependence of the prestretching force $F_{\mu}$ and the initial circumferential compression $\lambda_{\theta \Theta}{ }^{i n i}$ on the applied axial prestretch. The colours used to distinguish the individual prestretches are same in all figures and are chosen from HTML colour specification (in ascending order, they are: Black, Maroon, Red, DarkOrange, Gold, Yellow, GreenYellow, Cyan, DodgerBlue, Fuchsia, and DeepPink). 
The inflation-extension responses predicted by the system of equations (15-16) are depicted in $\mathrm{C}$ and $\mathrm{D}$ of Figure 1. It is clear that the circumferential distensibility, understood as $\lambda_{\theta \Theta}\left(P_{2}\right)-\lambda_{\theta \Theta}\left(P_{1}\right)$ for some fixed $\lambda_{z} z^{i n i}$ (where $P_{1}<P_{2}$ ), depends strongly on the chosen axial prestretch $\lambda_{z z^{i n i}}$. Consider e.g. the deep pink $\left(\lambda_{z} z^{i n i}=2\right)$ and black $\left(\lambda_{z} z^{i n i}=1\right)$ curves in panel C. It is clear that if $P_{\mu 2}=1$ and $P_{\mu 1}=0.5$, a greater stretch difference $\lambda_{\theta \Theta}\left(P_{\mu 2}\right)-\lambda_{\theta \Theta}\left(P_{\mu 1}\right)$ is obtained for greater prestretch.

This property is better documented in panels E and F. In E, the contours of the constant pressure $P_{\mu}=k$, where $k \in\{0.1(i-1)\}_{i=1}^{n=11}$, are added to the graph showing the inflation-extension responses as traces in the $\lambda_{\theta \Theta}-\lambda_{z Z}$ plane. Choosing again $\lambda_{z} z^{i n i}=1$ and 2 (black and deep pink solid curves), and for example $P_{\mu 2}=0.2$ and $P_{\mu 1}=0.1$ (red and maroon dashed curves), one can see that higher circumferential distensibility $\lambda_{\theta \Theta}\left(P_{\mu 2}\right)-\lambda_{\theta \Theta}\left(P_{\mu 1}\right)$ is obtained for $\lambda_{z} z^{i n i}=1$ (black solid). This demonstrates that the circumferential distensibility of the tube, $\lambda_{\theta \Theta}\left(P_{\mu 2}\right)-\lambda_{\theta \Theta}\left(P_{\mu 1}\right)$ (where $\left.P_{\mu 1}<P_{\mu 2}\right)$, is not monotonic with respect to the applied $\lambda_{z} z^{i n i}$ in the model based on (8).

Panel F displays this for pressures $P_{\mu 2}=P_{\mu}$ and $P_{\mu 1}=0$. In other words, panel F shows the difference between the circumferential stretch achieved at some pressure $P_{\mu}$ and the initial circumferential stretch that is ordinarily attained at $P_{\mu}=0$. It can be concluded that weakly prestretched tubes show mechanical responses with high circumferential distensibility at low pressures (consider e.g. the maroon $\sim \lambda_{z} z^{\text {ini }}=$ 1.1 , red $\sim \lambda_{z} z^{i n i}=1.2$, and dark orange $\sim \lambda_{z} z^{i n i}=1.3$ curves) in contrast to highly prestretched tubes. However, at higher pressures, the curves corresponding to higher prestretches exceed the curves obtained for less prestretched tubes (consider e.g. the curves in dodger blue $\sim \lambda_{z} z^{i n i}=1.8$, fuchsia $\sim \lambda_{z} z^{i n i}$ $=1.9$, and deep pink $\sim \lambda_{z} z^{i n i}=2$ ).

This implies that $\lambda_{\theta \Theta}-\lambda_{\theta \Theta}$ ini is not monotonous in its first derivatives with respect to $P_{\mu}$ for a given $\lambda_{z} z^{i n i}$. A consequence of this is the existence of inflection points where tangents to $\lambda_{\theta \Theta}-\lambda_{\theta \Theta}$ ini will have extremal slopes (see Figure $1 \mathrm{~F}$ ). Such a property, theoretically, allows tubes to be programmed to operate in an optimal working range (to optimize either the pressure difference for a given distensibility or the distensibility at some chosen pressure difference). We will not go into further details here. We have already found what we had been looking for: the positive effect of the axial prestretch is not restricted to anisotropy, as had been hypothesized in Horný et al. (2014b).

\subsection{RESULTS FOR A NEO-HOOKEAN TUBE AT FINITE STRAINS}

Figure 2 depicts the mechanical responses obtained for an incompressible neo-Hookean thin-walled tube at finite strains. The responses are governed by equations (25-26), and numerical simulation is again the only way to make a theoretical investigation of the effect of the prestretch. Figure 2 is fashioned in a similar way as Figure 1. Panels A and B show the initial conditions for inflation, C and D show the dimensionless pressure and the achieved stretches, and $\mathrm{E}$ and $\mathrm{F}$ again document the circumferential distensibility as such. 
In comparison with Figure 1, there is one substantial difference, which is indicated by the dotted parts of the curves. The dotted curves correspond to a loss of deformation stability. This phenomenon is well known to anyone who has ever inflated a party balloon (Chater and Hutchinson 1984; Gent 2005; Kanner and Horgan 2007; Gonçalves et al., 2008; Rodriguez and Merodio, 2011; Mao et al., 2014; Horný et al., 2015). The inflation instability is exhibited as a non-monotonic dependence of the inflation pressure on the circumferential stretch. At the point where stability is lost, increments in circumferential stretch are accompanied by decrements in applied pressure. This is exactly what happens with a party balloon - after some initial loading its response becomes unstable.

Since the onset of loss of stability is accompanied by buckling, which in the case of a cylindrical tube may appear as a bulge propagated in the axial direction, or as bending (resembling the deflection of a beam), or a bulge propagated in the radial direction, the assumptions contained in the geometrical equations (1) are violated in the subsequent inflation. In the absence of serious post-buckling analysis, results related to the post-buckling behaviour therefore have to be considered uncertain. However, this is beyond the scope of our present study. We will limit ourselves to the stable elastic response, which is indicated by the solid parts of the curves.

Panel $\mathrm{C}$ in Figure 2 shows that the applied axial prestretch (a) decreases the maximum pressure achievable in the deformation, and (b) also makes the tube more distensible in the circumferential direction. This is clear, when one considers the slopes of the tangents made to the pressure-stretch curves at any fixed pressure (Panel C). In other words, the pressure-circumferential stretch dependences in panel $\mathrm{C}$ form concave curves (under elastically stable deformations).

The same conclusion is obtained for panels E and F. Especially F, which, similarly to Figure 1, shows the difference between the circumferential stretch achieved by some pressure $P_{\mu}$ and the initial circumferential stretch obtained at $P_{\mu}=0$, clearly demonstrates that the higher the axial prestretch, the higher the circumferential distensibility at a given pressure.

Unlike for the strain-stiffening model (8), there is no violation of monotony. Considering equation (18), we see that both models have topredict the same mechanical behaviour whe $\beta \rightarrow 0$. Thisis also clear when the governing equations (15-16) and (25-26) are compared. Now we see that the existence of the non-monotonic effect of the axial prestretch (increased vs. decreased circumferential distensibility) in the Fung-Demiray model (8) is a consequence of the presence of material parameter $\beta$. $\beta$ contributes to the system by one additional degree of freedom, and allows a switch between the positive effect (enhancing the distensibility) and the negative effect (suppressing the distensibility) of prestretching that is exhibited by a tube while it is being inflated. 


\subsection{SECOND ORDER LINEAR ELASTICITY}

Figure 3 shows the results obtained for a linearized neo-Hookean material with the deformation described using the engineering strain tensor (36-37). It is depicted with solid curves and filled circles. The panels are again arranged in the same way as in Figures 1 and 2. However, for the sake of easy comparison, the results predicted using the finite strain neo-Hooke model are also displayed here; equations (38-39). They are indicated by dotted curves and empty circles. For the finite strain model, the results depicted in the figure were transformed from the original Green-Lagrange deformations to engineering strains according to the equation $\varepsilon k k=\sqrt{ }\left(1+2 E_{K K}\right)-1$, where $k=\theta, z$, and $K=\Theta, Z$. Thus the two models are displayed in the same quantities, which helps when comparing them, because the nonlinear effects are immediately clear when the results are displayed over coordinate axes scaled in infinitesimal strains.

The same axial prestrain sequences were applied in the linearized model (III) and in the in the finite strain model (II); $\left\{\varepsilon_{z} z^{i n i}\right\}_{j=1}{ }^{11}=\{0.02(j-1)\}_{j=1}{ }^{11}$. On the basis of the conclusion obtained using the totally linearized model (IV, equations 50-52), we know that in this case the system of governing equations gives linear pressure-deformation relationships. Hence, if the solid curves in Figure 3 deflect from (imaginary) straight lines, this is the effect of large displacements sustained by linear material. When the dotted curves are deflected from the solid curves, this is the effect of the incompressibility formulated in the finite strain description; compare equations (36-37) and (38-39).

The nonlinearity of the $P_{\mu}-\varepsilon_{\theta \theta}$ relationship is clear from Figure 3 (panel C), and occurs in both models. In contrast with panel $\mathrm{C}$, where both finite strain neo-Hooke and also linearized neo-Hooke with infinitesimal strains but large displacements show clear nonlinearity, $P_{\mu}-\varepsilon_{z z}$ relationships for the linearized model with the small strains but large displacements (solid curves) presented in panel D exhibit only limited deviations from straightness. Moreover, nonlinearity occurs where there are strains say $0.1<\varepsilon z z$. In this region, the results obtained with $\varepsilon$ used in the description have to be considered as estimates of reality, rather than as facts. However, the finite strain model displayed over the axis scaled in $\varepsilon_{z z}$ clearly deflects from straightness.

It is hard to draw conclusions about the circumferential distensibility on the basis of panel $\mathrm{C}$ of Figure 3. The effect of the prestretch is less clearly visible than in Figures 1 and 2. The stretch variation $\left|\varepsilon_{\theta \theta}-\varepsilon_{\theta \theta^{n i}}\right|$ is therefore depicted separately in Figure 4. In panel A, we observe that the curves are convex. This indicates that distensibility increases with increasing pressure. The effect of the prestretch is positive; that is the greater the prestretch, the greater the distensibility. This is found by comparing the mutual positions of the curves (panel B). However, in the range of linear elasticity, this effect is almost negligible. 


\subsection{FIRST ORDER LINEAR ELASTICITY}

Total linearization (small strains and displacements) is the only case where conclusions can be drawn immediately on the basis of the equations (50-52). There is no effect of the axial prestrain on the mechanical response of an incompressible linearly elastic thin-walled tube apart from the shift of the linear $P_{\mu}-\varepsilon_{\theta \theta}$ relationship. There is no enhancement or suppression of circumferential distensibility, as is documented in Figure 5.

However, from a different point of view, insensitivity of the circumferential distensibility in the first order linear elasticity to the axial prestrain elucidates a source of the phenomenon under discussion here. The difference between first order and second order linear elasticity consists in the form of the right side in the equilibrium equations (48-49) vs. (36-37); i.e. in the nominal stress tensor (current force per reference cross-section, 48-49) vs. the Cauchy stress tensor (current force per deformed cross-section, 36-37). In other words, the reason for the enhanced distensibility in the second order linear theory lies in the large displacements. However, the comparison in Figure 3 shows that a finite strain formulation of the incompressibility moves the effect of the prestretch from a rather abstract mathematical phenomenon to a fact measurable by engineering methods.

\section{Conclusion}

The study published by Horný et al. (2014b) showed that nonlinear incompressible anisotropic thinwalled tubes exhibit higher circumferential distensibility when they are axially prestretched than nonprestretched tubes with the same constitutive properties and geometry. From a physiological point of view, this effect is positive because aortas, which were investigated in Horný et al. (2014b), serve as conduits for flowing blood. Thus the greater the distension that they are capable of achieving at some pressure, the larger is the volume of the blood that they can accommodate. Horný et al. (2014b) hypothesized that this effect, which had not been described previously in the literature, could be a consequence of arterial anisotropy.

In the present study, we have tried to show the true physical cause of the increased distensibility of axially prestretched tubes. The approach that has been adopted is based on a mutual comparison of four computational models. To be more specific, our study has investigated the mechanical response of a thin-walled incompressible tube (I) with a material based on the exponential strain energy density function, (II) with a neo-Hooke material, (III) with a linearly elastic material sustaining small strains but large displacements, and (IV) a tube with a totally linearized material. All the material models were isotropic.

The simulations showed that the positive effect of axial prestretching is not a property exclusively related to anisotropy, because the results obtained in (I) showed that axially prestretched tubes can distend more than non-prestretched tubes. The Fung-Demiray constitutive model used in case (I) is a direct isotropic restriction of the model used by Horný et al. (2014b). 
It has been proved that nonlinear effects are crucial for the positive role of axial prestretching in pressurization. Nonlinear constitutive models depending on more than one parameter (exemplified here by the Fung-Demiray model in I) can exhibit both enhancement and suppression of the circumferential distensibility of the tube, due to prestretching. This implies that the effect of prestretching in two or more parametrical nonlinear constitutive models can be positive (higher circumferential distensibility) or negative (lower distensibility relative to the response of a non-prestretched tube), and the specific result depends on the constitutive model and the pressure that is applied. By contrast, the one-parameter nonlinear model (II, neo-Hooke) showed only increased distensibility when axial prestretching had been applied.

A reduction of the computational model to second order linear elasticity (III, small strains but large displacements) led to mechanical responses that exhibited only a slight effect of prestretching in comparison with previous nonlinear models. However, from the purely mathematical point of view, the positive effect of prestretching on circumferential distensibility is still present. In case (III), highly prestretched tubes showed higher distensibility than weakly prestretched tubes.

Finally, total linearization (IV) proved that the significant effect is present only to the point at which the deformed configuration and the reference configuration are considered to be different. In other words, first order linear elasticity (IV), which does not distinguish between the two configurations when the stresses are computed from the loads applied to a structure, showed no other effect of prestretching apart from a change in the initial conditions of the pressurization. Neither enhancement nor suppression of the circumferential distensibility was found.

To the best of our knowledge, this is the first study that has made a systematic evaluation of the effect of prestretching on the mechanical response of pressurized nonlinear tubes. However, this does not mean that there have been no previous papers documenting our results. As is shown in Horný et al. (2014b), there have been studies documenting experimentally that arteries pressurized ex vivo exhibit higher circumferential distensibility when they are axially prestretched (cf. Figure 4 in Schulze-Bauer et al., 2003; Figure 5 in Sommer et al., 2010; Figure 4 in Sommer and Holzapfel 2012; Figure 6 in Avril et al., 2013). However, a detailed discussion of this phenomenon was not an objective of these papers. Details of the physiological and mechanobiological role of prestretching can be found in Humphrey et al. (2009) and Cardamone et al. (2009).

Finally it should be noted explicitly that first order linear theory is a limit of all nonlinear theories. Hence, irrespective of the formalism (nonlinear theory, a linear material for finite strains, a linear material under large displacements but small strains), if the displacements and strains are sufficiently small, the results obtained with first order linear elasticity will also hold for other formalisms. In other words, one when chooses some small positive epsilon as the error between linear and nonlinear predictions, there will always be some delta bordering a subset in the space of deformations where the errors between linear and nonlinear theory will be smaller than the chosen epsilon. In engineering practice, epsilon depends on the sensitivity and the confidence of our experimental methods. 


\section{ACKNOWLEDGEMENT}

L.H. has been supported in the project of the Czech Ministry of Health NT 13302, and M.N. was supported by the Faculty of Mathematics and Physics of Charles University in Prague in project no. $260220 / 2015$.

\section{REFERENCES}

Avril, S., Badel, P., Gabr, M., Sutton, M. A., Lessner, S. M., 2013. Biomechanics of porcine renal arteries and role of axial stretch. Journal of Biomechanical Engineering, 135(8), art. no. 081007.

Bergel, D.H., 1961. The static elastic properties of the arterial wall. J. Physiol. 156, 445-457.

Cardamone, L., Valentín, A., Eberth, J. F., Humphrey, J. D., 2009. Origin of axial prestretch and residual stress in arteries. Biomechanics and Modeling in Mechanobiology, 8(6), 431-446.

Chater, E., Hutchinson, J.W., 1984. On the propagation of bulges and buckles. J. Appl. Mech. Trans. ASME 51, 269-277.

Demiray, H., 1972. A note on the elasticity of soft biological tissues. J. Biomech. 5, 309-311.

Dobrin, P.B., Doyle, J.M., 1970. Vascular smooth muscle and the anisotropy of dog carotid artery. Circ. Res. 27, 105-119.

Fuchs, R.F., 1900. Zur Physiologie und Wachstumsmechanik des Blutgefass-systems. Arch. Anat. Physiol. 102-154.

Fung, Y.C. 1967. Elasticity of soft tissues in simple elongation. Am. J. Physiol. 213, 1532-1544.

Fung, Y.C., Fronek, K., Patitucci, P., 1979. Pseudoelasticity of arteries and the choice of its mathematical expression. Am. J. Physiol. 237, H620-631.

Fung, Y.C., 1990. Biomechanics: Motion, Flow, Stress, and Growth. Springer-Verlag, New York.

Fung, Y.C., 1997. Biomechanics: Circulation. Springer-Verlag, New York, second edition.

Gasser, T.C., Ogden, R.W., Holzapfel, G.A., 2006. Hyperelastic modelling of arterial layers with distributed collagen fibre orientations. J. R. Soc. Interface 3, 15-35.

Gent, A.N., 2005. Elastic instabilities in rubber. Int. J. Non-Lin. Mech. 40, 165-175.

Gonçalves, P.B., Pamplona, D., Lopes, S.R.X., 2008. Finite deformations of an initially stressed cylindrical shell under internal pressure. Int. J. Mech. Sci. 50, 92-103.

Guccione, J.M., McCulloch, A.D., Waldman, L.K. 1991. Passive material properties of intact ventricular myocardium determined from a cylindrical model. J. Biomech. Eng. 113, 42-55.

Holzapfel, G.A., 2000. Nonlinear solid mechanics: A continuum approach for engineering. John Wiley and Sons, Chichester.

Holzapfel, G.A., Gasser, T.C., Ogden, R.W., 2000. A new constitutive framework for arterial wall mechanics and a comparative study of material models. J. Elast. 61, 1-48.

Holzapfel, G.A., Ogden, R.W., 2010. Constitutive modelling of arteries. Proc. R. Soc. A-Math. Phys. Eng. Sci. 466, 1551-1597.

Horgan, C.O., Saccomandi, G., 2003. A description of arterial wall mechanics using limiting chain extensibility constitutive models. Biomech. Model. Mechanobiol. 1, 251-266.

Horgan, C.O., 2015. The remarkable Gent constitutive model for hyperelastic materials. Int. J. Non-Lin. Mech. 68, 9-16. 
Horny, L., Hulan, M., Zitny, R., Chlup, H., Konvickova, S., Adamek, T., 2009. Computer-aided analysis of arterial wall architecture. IFMBE Proceedings, 25(4):1494-1497.

Horny, L., Kronek, J., Chlup, H., Zitny, R., Vesely, J., Hulan, M., 2010. Orientations of collagen fibers in aortic histological section. B. Appl. Mech. 6, 25-29.

Horný, L., Adámek, T., Gultová, E., Žitný, R., Veselý, J., Chlup, H., Konvičková, S., 2011. Correlations between age, prestrain, diameter and atherosclerosis in the male abdominal aorta. J. Mechan. Behav. Biomed. Mater. 4, 2128-2132.

Horny, L., Adamek, T., Vesely, J., Chlup, H., Zitny, R., Konvickova, S., 2012. Age-related distribution of longitudinal pre-strain in abdominal aorta with emphasis on forensic application. Forensic Science International, 214(1-3), 18-22.

Horny, L., Adamek, T., Chlup, H., Zitny, R., 2012. Age estimation based on a combined arteriosclerotic index. International Journal of Legal Medicine, 126(2), 321-326.

Horný, L., Adámek, T., Žitný, R., 2013. Age-related changes in longitudinal prestress in human abdominal aorta. Arch. Appl. Mech. 83, 875-888.

Horny, L., Adamek, T., Kulvajtova, M., 2014a. Analysis of axial prestretch in the abdominal aorta with reference to post mortem interval and degree of atherosclerosis. J. Mechan. Behav. Biomed. Mater. 33, 93-98.

Horný, L., Netušil, M., Voňavková, T., 2014b. Axial prestretch and circumferential distensibility in biomechanics of abdominal aorta. Biomech. Model. Mechanobiol. 13, 783-799.

Horný, L., Netušil, M., Daniel, M., 2014c. Limiting extensibility constitutive model with distributed fibre orientations and ageing of abdominal aorta. J. Mechan. Behav. Biomed. Mater. 38, 39-51.

Horný, L., Netušil, M., Horák, Z., 2015. Limit point instability in pressurization of anisotropic finitely extensible hyperelastic thin-walled tube. Int. J. Non-Lin. Mech., article in press. DOI: 10.1016/j.ijnonlinmec.2015.08.003

Humphrey, J.D., 2002. Cardiovascular Solid Mechanics: Cells, Tissues, and Organs. Springer-Verlag, New York.

Humphrey, J. D., Eberth, J. F., Dye, W. W., Gleason, R. L., 2009. Fundamental role of axial stress in compensatory adaptations by arteries. Journal of Biomechanics, 42(1), 1-8.

Kanner, L.M., Horgan, C.O., 2007. Elastic instabilities for strain-stiffening rubber-like spherical and cylindrical thin shells under inflation. Int. J. Non-Lin. Mech. 42, 204-215.

Mao, G., Li, T., Zou, Z., Qu, S., Shi, M., 2014. Prestretch effect on snap-through instability of shortlength tubular elastomeric balloons under inflation. Int. J. Solids Struct. 51, 2109-2115.

Ogden, R.W., Saccomandi, G., 2007. Introducing mesoscopic information into constitutive equations for arterial walls. Biomech. Model. Mechanobiol. 6, 333-344.

Rodríguez J., Merodio, J., 2011. A new derivation of the bifurcation conditions of inflated cylindrical membranes of elastic material under axial loading: application to aneurysm formation. Mech. Res. Commun. 38, 203-210.

Schulze-Bauer, C.A.J., Mörth, C., Holzapfel, G.A., 2003. Passive biaxial mechanical response of aged human iliac arteries. J. Biomech. Eng. 125, 395-406.

Sommer, G., Regitnig, P., Költringer, L., Holzapfel, G.A., 2010. Biaxial mechanical properties of intact and layer-dissected human carotid arteries at physiological and supraphysiological loadings. Am. J. Physiol. - Heart Circ. Physiol. 298, H898-H912. 
Sommer, G., Holzapfel, G. A., 2012. 3D constitutive modeling of the biaxial mechanical response of intact and layer-dissected human carotid arteries. Journal of the Mechanical Behavior of Biomedical Materials, 5(1), 116-128.

Taber, L.A., 2004. Nonlinear Theory of Elasticity: Applications in Biomechanics. World Scientific Publishing, New Jersey.

Van Loon, P., Klip, W., Bradley, E.L., 1977. Length-force and volume-pressure relationships of arteries. Biorheology 14, 181-201.

\section{LEGENDS OF FIGURES}



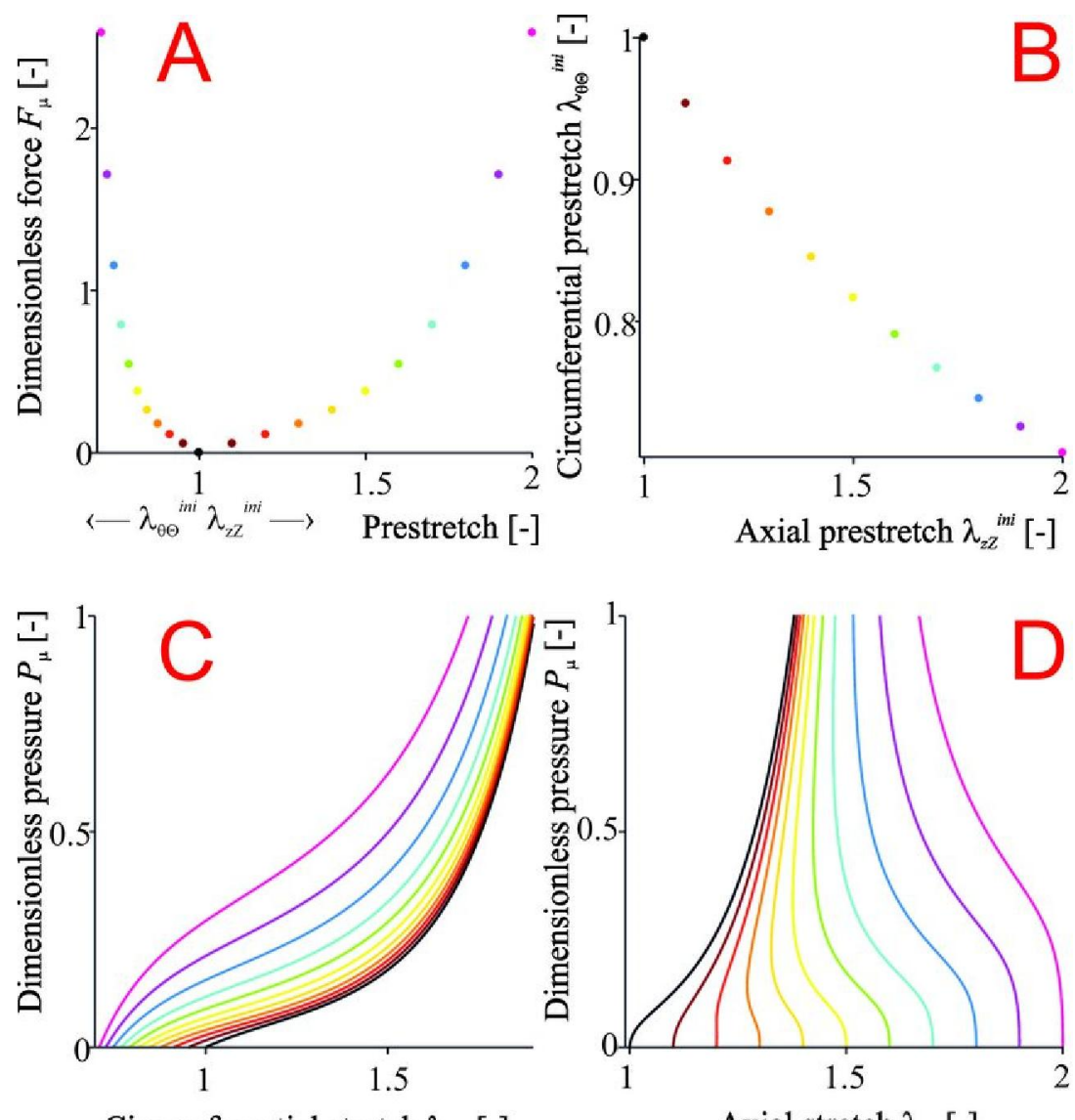

Circumferential stretch $\lambda_{\theta \Theta}[-]$

Axial stretch $\lambda_{z z}[-]$

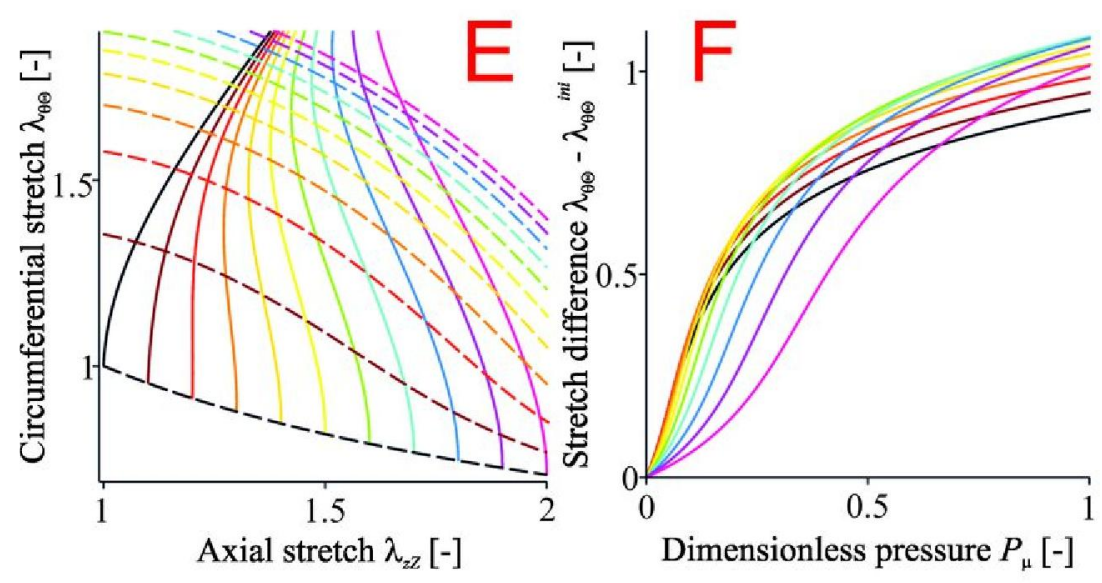

Axial prestretch

$$
\begin{gathered}
\lambda_{z z}^{\text {ini }} \in\{1,1.1,1.2,1.3,1.4,1.5,1.6,1.7,1.8,1.9,2\} \\
P_{\mu}=k \text { contours in } \lambda_{z z}-\lambda_{\text {өө }} \\
k \in\{0,0.1,0.2,0.3,0.4,0.5,0.6,0.7,0.8,0.9,1\}
\end{gathered}
$$

Figure 1. Fung-Demiray inflation-extension response. A - initial prestretch and dimensionless force. $\mathrm{B}-$ mutual dependence of initial prestretches $\left(\lambda_{z} z^{i n i}-\lambda_{\theta \Theta^{i n i}}\right)$. C and D - dimensionless pressure vs. stretch. E - traces of the inflation-extension responses in the phase space of the deformation (solid curves) and contour curves for dimensionless pressure $P_{\mu}=k$ (dashed curves). F - stretch difference $\lambda_{\theta \Theta}$ $-\lambda_{\theta \Theta}{ }^{i n i}$ achieved by loading a tube with pressure $P_{\mu}$. 

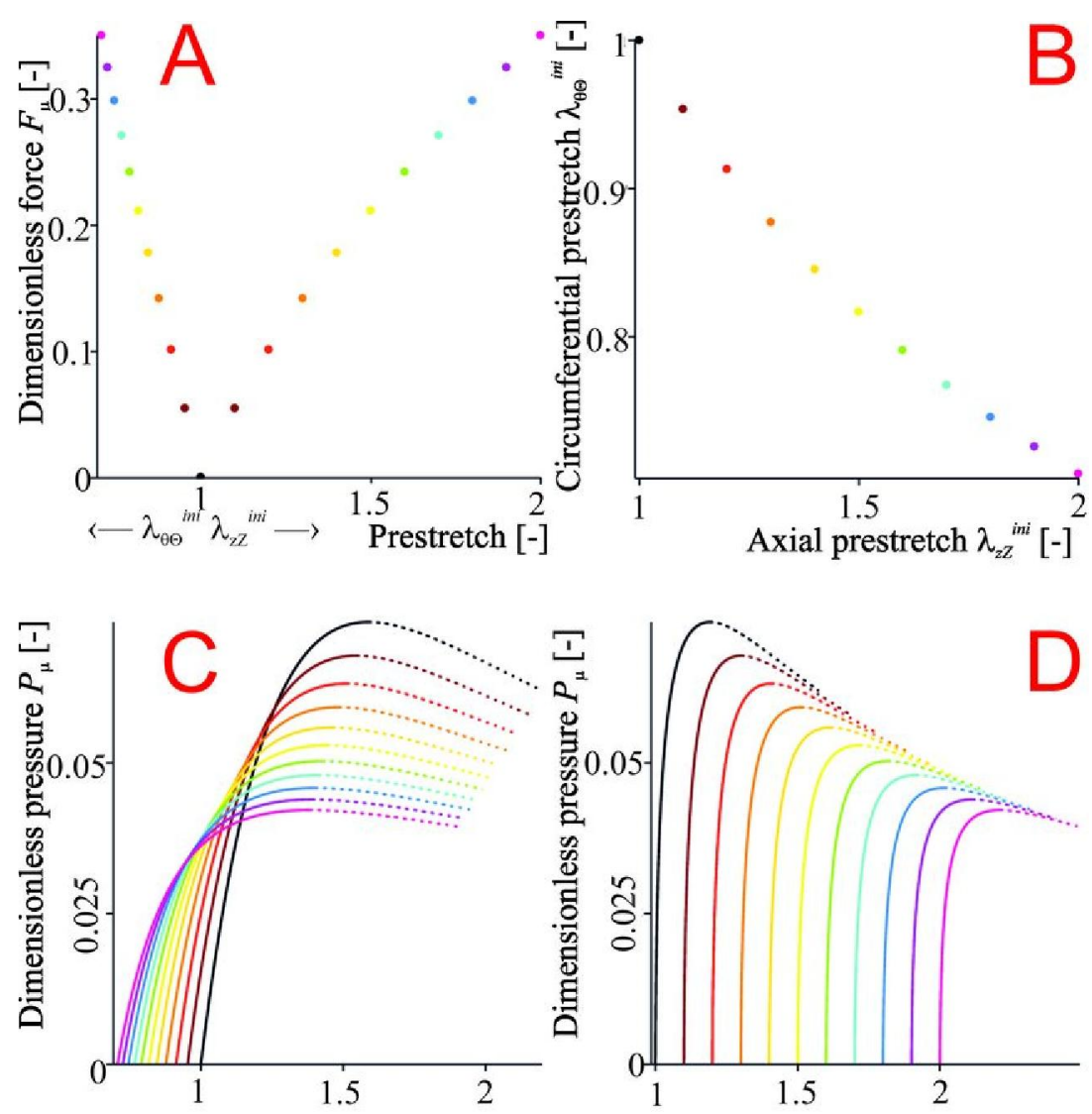

Circumferential stretch $\lambda_{\theta \Theta}[-]$

Axial stretch $\lambda_{z Z}[-]$
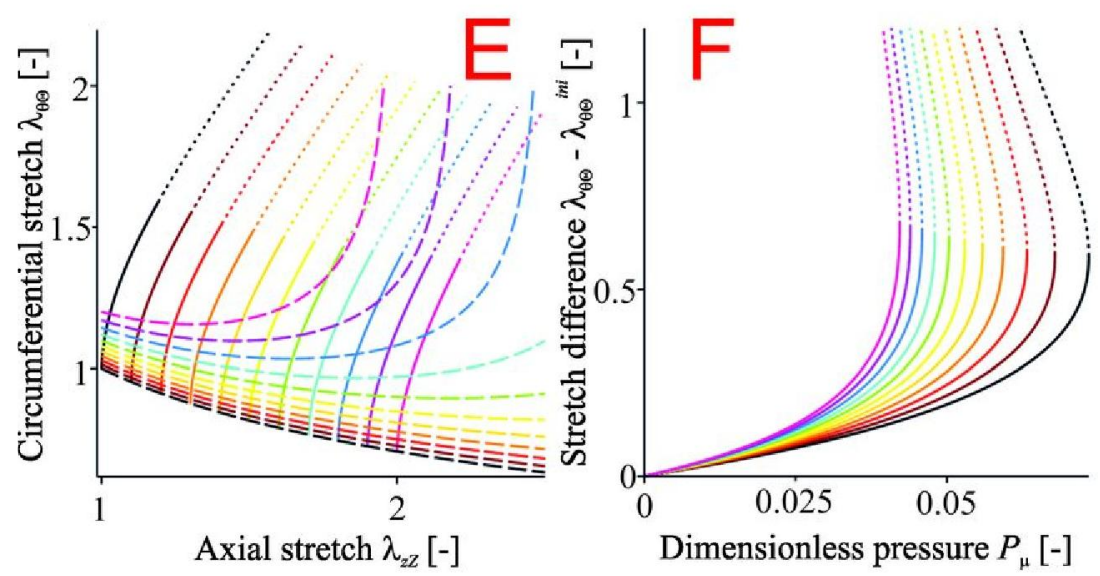

Axial prestretch

$$
\begin{gathered}
\lambda_{z Z}{ }^{i n i} \in\{1,1.1,1.2,1.3,1.4,1.5,1.6,1.7,1.8,1.9,2\} \\
P_{\mu}=k \text { contours in } \lambda_{z Z}-\lambda_{\theta \oplus} \\
k \in\{0,0.005,0.01,0.015,0.02,0.025, \\
0.03,0.035,0.04,0.045,0.05\}
\end{gathered}
$$

Figure 2. Neo-Hookean inflation-extension response. A - initial prestretches and dimensionless force. $\mathrm{B}-$ mutual dependence of the initial prestretches $\left(\lambda_{z} z^{i n i}-\lambda_{\theta \Theta}{ }^{i n i}\right)$. C and D - dimensionless pressure vs. stretch. E - traces of the inflation-extension responses in the phase space of the deformation (solid curves) and contour curves for dimensionless pressure $P_{\mu}=k$ (dashed curves). $\mathrm{F}-$ stretch difference $\lambda_{\theta \Theta}$ $-\lambda_{\theta \Theta}{ }^{i n i}$ achieved by loading a tube with pressure $P_{\mu}$. Dotted curves indicate a loss of deformation stability. 

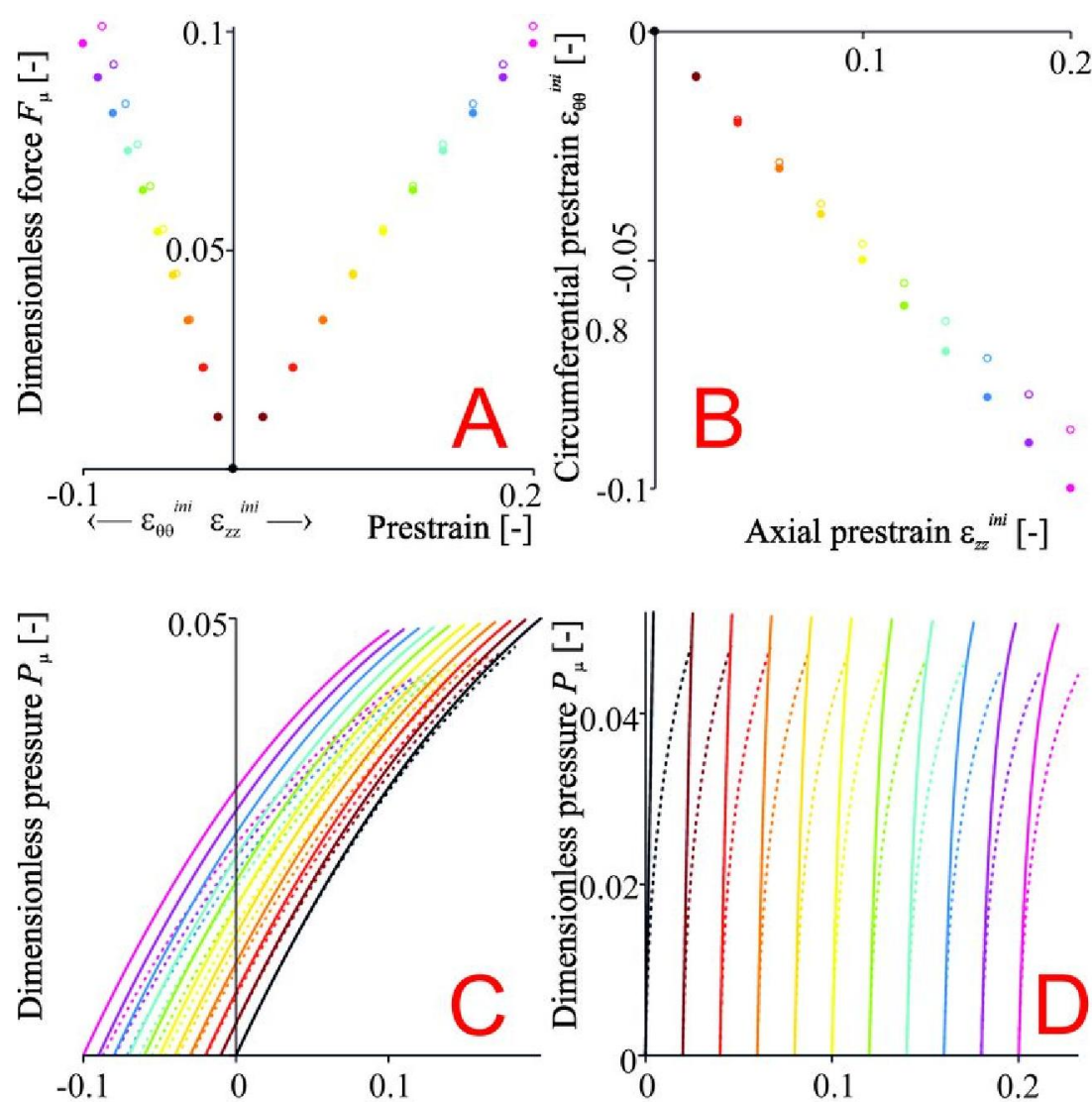

Circumferential strain $\varepsilon_{\theta \theta}[-]$
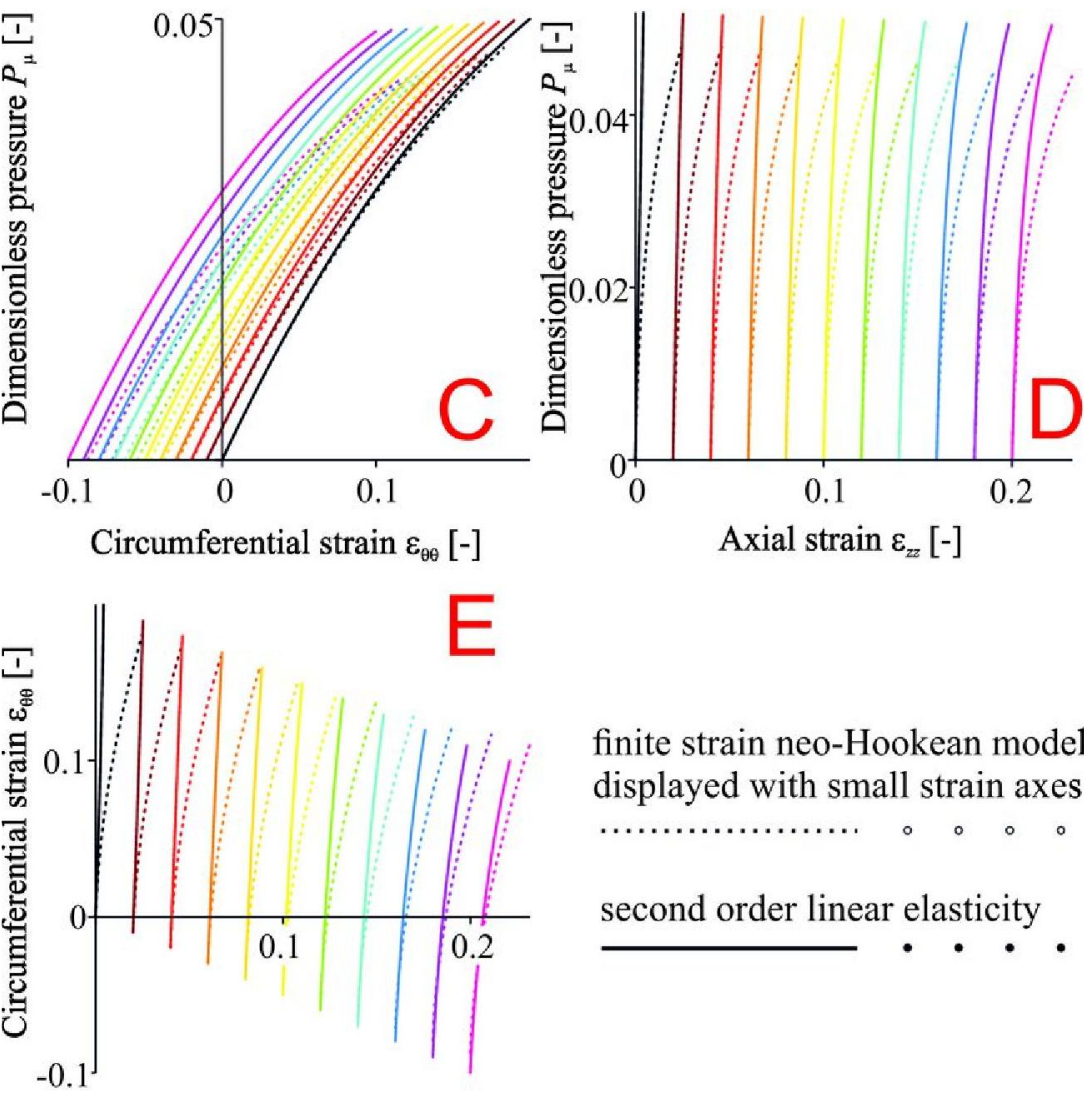

Axial strain $\varepsilon_{z z}[-]$

Axial strain $\varepsilon_{z z}[-]$

\section{Axial prestrain}

$\varepsilon_{z z}^{i n i} \in\{0,0.02,0.04,0.06,0.08,0.1,0.12,0.14,0.16,0.18,0.2\}$

Figure 3. The neo-Hookean and linearized inflation-extension response at small strains but large displacements. A - prestrains and dimensionless force. B - mutual dependence of initial prestrains. $\mathrm{C}$ and $\mathrm{D}$ - dimensionless pressure vs. infinitesimal strain. $\mathrm{E}$ - traces of inflation-extension responses. The solid circles and continuous curves correspond to second order linear theory. The dotted curves and empty circles correspond to nonlinear theory applied to a neo-Hookean material, but the results are displayed over infinitesimal strain coordinates according to $\varepsilon k k=\sqrt{ }\left(1+2 E_{K K}\right)-1$. 

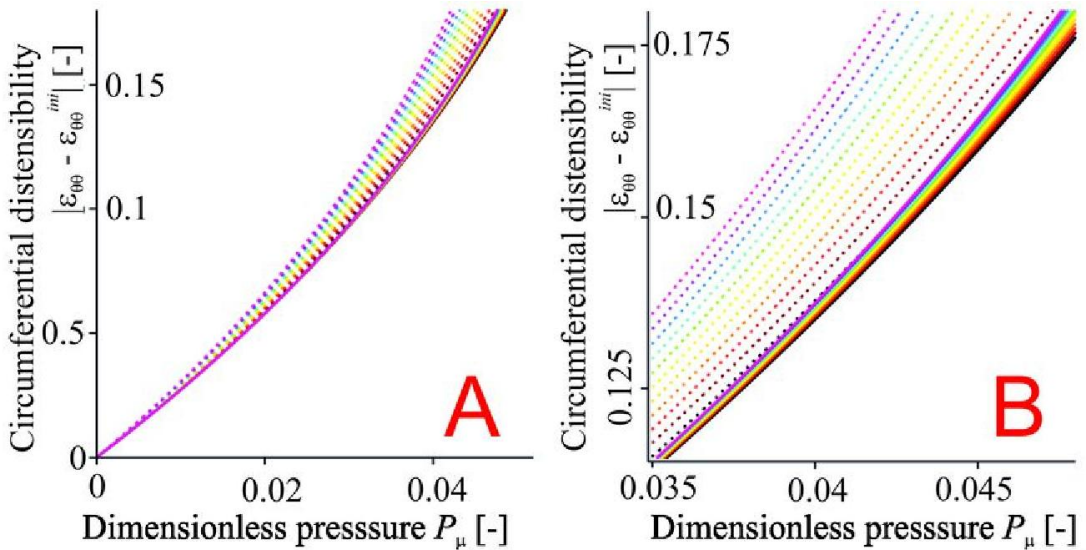

Axial prestrain

$$
\begin{aligned}
& \varepsilon_{z z}^{\text {ini }} \in\{0,0.02,0.04,0.06,0.08,0.1,0.12,0.14,0.16,0.18,0.2\} \\
& \text { finite strain neo-Hookean model } \\
& \text { displayed with small strain axes } \\
& \text { - second order linear elasticity }
\end{aligned}
$$

Figure 4. Circumferential distensibility of the Neo-Hookean model and the linearized model in the inflation-extension response at small strains but large displacements. A - overall dependences. B detail.
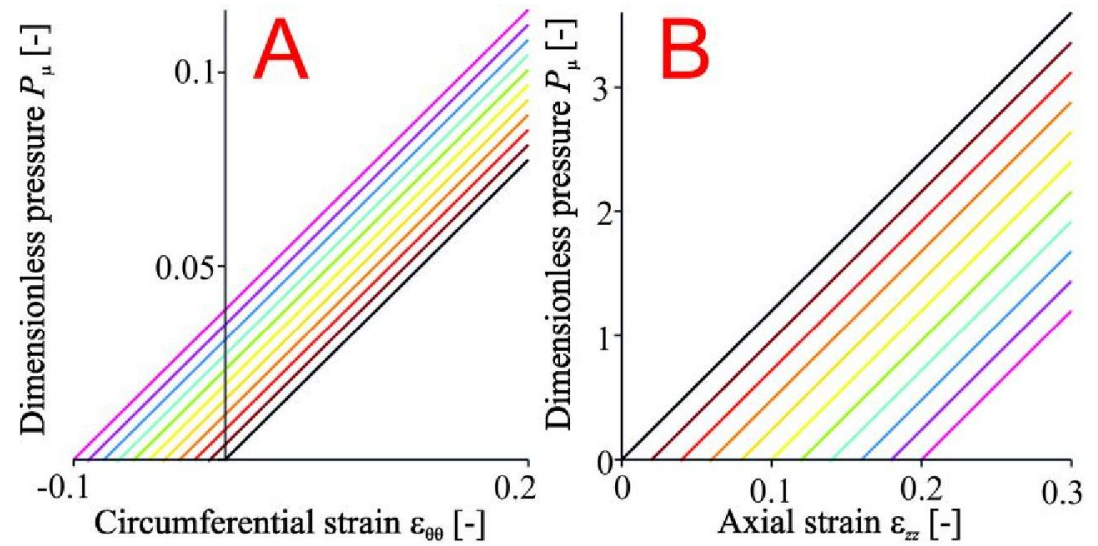

Axial prestrain

$\varepsilon_{z z}^{\text {ini }} \in\{0,0.02,0.04,0.06,0.08,0.1,0.12,0.14,0.16,0.18,0.2\}$

Figure 5. Inflation-extension responses in the first order linear elasticity. A - circumferential responses. $\mathrm{B}$ - longitudinal responses. 